\title{
LMTK3 inhibition affects microtubule stability
}

\author{
Chiara Cilibrasi', Angeliki Ditsiou', Athanasios Papakyriakou², George Mavridis², Murat Eravci' , Justin Stebbing ${ }^{3}$, \\ Teresa Gagliano ${ }^{1,4}$ and Georgios Giamas ${ }^{1 *}$ (D)
}

Keywords: LMTK3, Kinase inhibitor, Breast cancer, Tubulin, NUSAP1

\section{Results and discussion}

C28 induces mitotic arrest in breast cancer cells

Human lemur tyrosine kinase 3 (LMTK3) is a dual specificity kinase, whose oncogenic role has been wellestablished in different tumour types, supporting its potential as a therapeutic target [1]. Recently, using robust in vitro and cell-based screening- and selectivityassays combined with biophysical analyses, we identified a selective small molecule ATP-competitive LMTK3 inhibitor (C28; Fig. 1a) that acts by degrading LMTK3 via the ubiquitin-proteasome pathway. C28 demonstrated effective anticancer effects in a variety of cancer cell lines and in in vivo breast cancer (BC) mouse models [2]. This potent, selective and cell-permeable inhibitor represents an effective tool to investigate and decipher the signalling pathways in which LMTK3 is implicated and progress with our translational research work.

In order to decipher the mechanism of action of C28, we initially performed flow cytometry (FACS) analysis and revealed that treatment with $\mathrm{C} 28$ leads to $\mathrm{G} 2 / \mathrm{M}$ phase arrest of BC cells as well as MCF12A, a nontransformed breast cell line (Fig. 1b). This result was confirmed by evaluating the increased mitotic index (Fig. 1c) and by the upregulation of the mitotic marker phospho-histone H3 (Ser10) (Fig. 1d). As previously reported [2], prolonged exposure to C28 induced apoptosis of $\mathrm{BC}$ cells but had a low $\%$ of cell death $(<5 \%)$ in

\footnotetext{
* Correspondence: g.giamas@sussex.ac.uk

${ }^{1}$ Department of Biochemistry and Biomedicine, School of Life Sciences, University of Sussex, JMS Building, Falmer, Brighton BN1 9QG, UK Full list of author information is available at the end of the article
}

MCF12A, suggesting that non-transformed breast epithelial cells evade death while working on recuperating from cell cycle arrest (Fig. 1b). Similarly, silencing of LMTK3 led to an increase in phospho-histone H3 (Ser10) levels, suggesting a potential role of LMTK3 in G2/M transition (Fig. 1e), although no evident effects on cell cycle distribution were observed during the $72 \mathrm{~h}$ period of siRNA treatment (data not shown), in accordance with previously published data [1].

\section{C28 interferes with tubulin polymerization and mitotic spindle organization}

Bearing in mind the effects of C28 on cell cycle arrest and the induction of cell death, we investigated whether C28 impacts microtubule dynamics, which can in turn disturb the organization of the cytoskeleton and affect cell division [3]. Immunofluorescence of cells at the metaphase revealed that C28-treated cells present disrupted microtubule distribution and mitotic defects, including abnormal microtubule spindle organization and an altered chromosome condensation pattern (Supplementary Fig. S1a).

Following, the effect of $\mathrm{C} 28$ on microtubule stability was confirmed using a cell-based microtubule polymerization assay, where we observed a dose dependent decrease of the insoluble polymerized tubulin fraction. (Fig. 1f). Interestingly, the magnitude of $\mathrm{C} 28$ effects appeared to be cellline dependent, suggesting the existence of different proteins and signalling pathways that may be implicated and affect this phenotype.

Considering the role of kinase inhibitors on microtubules $[4,5]$, we investigated the possibility that C28 is a 


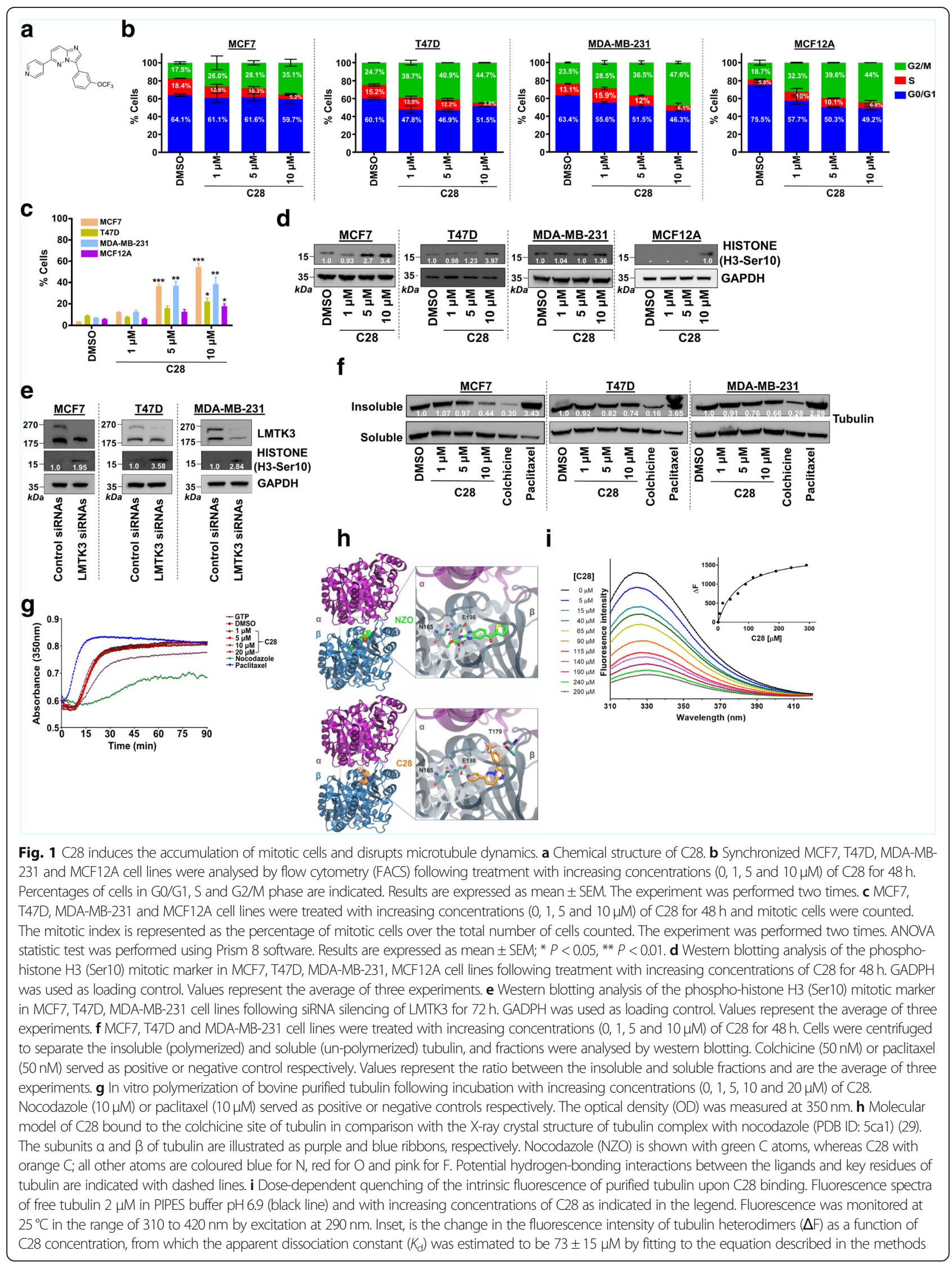


direct tubulin-targeting agent by employing an in vitro tubulin polymerization assay. The effects on the assembly of purified porcine tubulin were evaluated by measuring the absorbance at $350 \mathrm{~nm}$ at $37^{\circ} \mathrm{C}$, using paclitaxel and nocodazole as comparative agents. As expected, paclitaxel promoted tubulin polymerization, while nocodazole inhibited it, as demonstrated by the increased fluorescence intensity (compared to the control) in the former and decrease in the latter. Contrariwise, incubation of tubulin with increasing concentrations of C28 at different time points had no effects on tubulin polymerization (Fig. 1g).

To examine whether $\mathrm{C} 28$ binds tubulin at the atomic level, we performed docking calculations of $\mathrm{C} 28$ in comparison with nocodazole, a reversible inhibitor of microtubule polymerization and a high-affinity ligand for the cancer-related kinases ABL, BRAF, c-KIT and MEK [6]. Our docking results using the high-resolution X-ray crystal structure of tubulin complex with nocodazole (PDB ID: $5 \mathrm{ca} 1)$ revealed that $\mathrm{C} 28$ can be accommodated at the colchicine binding site of the $\beta$-subunit of tubulin (Fig. 1h). In particular, C28 is predicted to interact with both $\alpha$ and $\beta$ subunits of the curved (unassembled) tubulin, but not as deep inside the $\beta$ subunit as nocodazole. Compared with nocodazole that has been shown to bind $\beta$-tubulin via hydrogen-bonding interactions with Asn165 and Glu198 (Fig. 1h), C28 displayed a potential hydrogen bonding interaction with Glu198 and a halogen bond with Thr179 of the $\alpha$-subunit. The free energy of binding to tubulin was estimated to be $-9.1 \mathrm{kcal} / \mathrm{mol}$ for $\mathrm{C} 28$ and $10.4 \mathrm{kcal} / \mathrm{mol}$ for nocodazole, suggesting an approximately 10 -fold lower affinity of C28 for tubulin.

The binding affinity of $\mathrm{C} 28$ to tubulin was further examined in vitro by monitoring the intrinsic tryptophan fluorescence of tubulin, a widely used method to determine the binding affinity of drugs for tubulin heterodimers [7]. Incubation of purified porcine tubulin with $\mathrm{C} 28$ revealed a concentration-dependent quenching of the fluorescence at $310-420 \mathrm{~nm}$ and the changes in the fluorescence intensity were fitted to a binding isotherm as previously described [7] (Fig. 1i). The apparent dissociation constant $\left(K_{\mathrm{d}}\right)$ of $\mathrm{C} 28$ to purified tubulin heterodimers was estimated to be $73 \pm 15 \mu \mathrm{M}$. For comparison, the binding affinity of nocodazole for purified tubulin was measured with apparent dissociation constants between 0.29 and $1.54 \mu \mathrm{M}$, depending on the specific tubulin isotype used. This result is in agreement with the lack of any observable effect on tubulin polymerization upon treatment with $\mathrm{C} 28$ concentrations of $1-20 \mu \mathrm{M}$ in vitro (Fig. $1 \mathrm{~g}$ ).

Taken together, although C28 can potentially bind tubulin, its relatively low binding affinity to purified tubulin dimer and the lack of any observable effect on tubulin polymerization suggest that $\mathrm{C} 28$ does not confer its effects by direct inhibition of tubulin polymerization. Instead our data imply that C28 modulates LMTK3-regulated pathways linked to microtubule assembly, a result that can partly explain the previously observed universal cytotoxic effects of C28 on the NCI-60 cancer cell line panel [2].

\section{Pharmacological or genetic inhibition of LMTK3 downregulates NUSAP1 microtubule-associated protein}

In an attempt to decipher the signalling pathways via which C28 confers its effects on microtubules assembly, we used a tandem mass tagging (TMT) quantitative proteomic approach and uncovered the C28-mediated global proteomic alterations in BC cells (Fig. 2a). Following quantile normalization of TMT label intensities for each channel, 2852 distinguishable and unambiguous proteins were identified with a minimum of one unique peptide with a false discovery rate (FDR) of 1\% (Supplementary Table S1). As anticipated, LMTK3 was amongst the downregulated proteins following treatment with C28 (Fig. 2a).

Interestingly, amongst the most significant C28modulated hits was NUSAP1 $(P<0.05$ and Log2 fold change of $\geq|1.25|$ ), a microtubule associated protein participating in mitotic spindle organization $[8,9]$, whose role has been closely associated with tumour progression, chemoresistance, and poor prognosis in many tumours including $B C[10,11]$. The effects of C28 on NUSAP1 were validated in additional $\mathrm{BC}$ cell lines (Fig. $2 \mathrm{~b}$ ). In addition, to confirm the involvement of NUSAP1 in the C28mediated effect on microtubule stability and cell cycle arrest, we investigated the levels of cyclin-dependent kinase 1 (CDK1), a previously described NUSAP1-regulated protein [12], and phospho-BIII tubulin (S172). This latter phosphorylation is catalysed by CDK1 and has been shown to influence microtubule dynamics by affecting their polymerization [13, 14] (Fig. 2b) To corroborate that the observed effects are due to the selective C28-mediated LMTK3 inhibition, we silenced LMTK3 (siRNA) and saw a downregulation of NUSAP1, CDK1, and phospho-BIII tubulin (S172) (Fig. 2c). To further establish that this was an LMTK3/NUSAP1-mediated effect, we restored LMTK3 levels following C28 treatment and detected a rescue of NUSAP1 levels (Fig. 2d). In addition, a recovery of CDK1, phospho-BIII tubulin (S172) and phosphohistone H3 (Ser10) levels were observed after restoring NUSAP1 following C28 treatment (Fig. 2e). Altogether, these data confirm that $\mathrm{C} 28$ affects microtubule dynamics via an LMTK3 and NUSAP1-regulated pathway. Moreover, following immunoprecipitation experiments, an endogenous interaction of LMTK3 with NUSAP1 was discovered (Fig. 2f), suggesting, as previously reported [15], that LMTK3 may function as a scaffold protein able to interact with and stabilize NUSAP1.

Finally, we investigated the association between mRNA levels of LMTK3 and NUSAP1 with survival. Our analysis revealed that high expression of LMTK3 and 


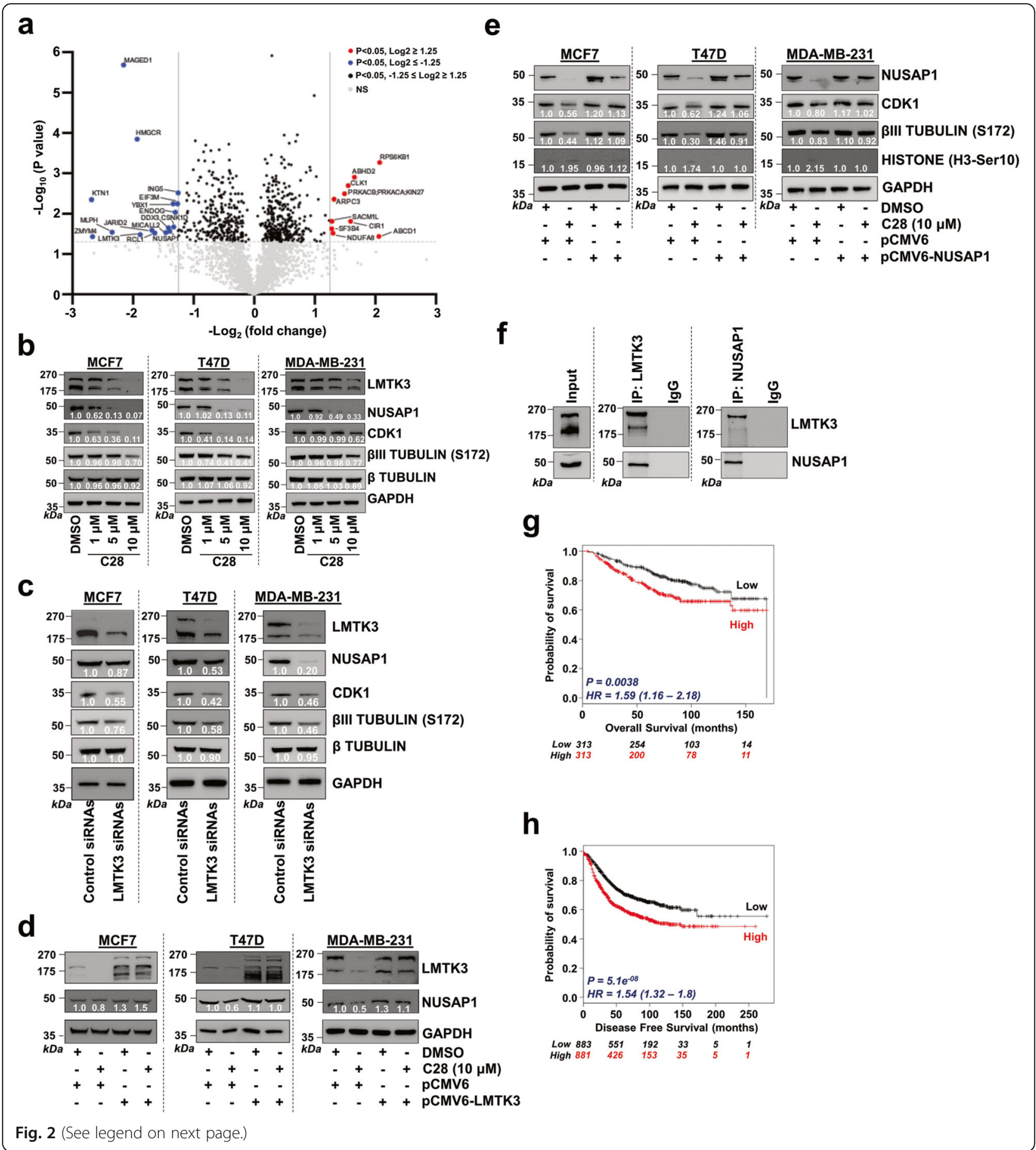


(See figure on previous page.)

Fig. 2 C28 decreases NUSAP1 protein levels. a Volcano plot of differentially expressed proteins following treatment with C28 in MCF7 cells stably overexpressing LMTK3. The plot illustrates the - $\log _{10} P$-value vs. the $\log _{2}$ fold change of protein abundance in the presence of $C 28$. The significance threshold $(P=0.05)$ is represented by a horizontal line. The two vertical lines (Log2 fold change of $\geq 1.5$ and $\leq-1.5)$ represent the cut-off values of interest. $\mathbf{b}$ Western blotting analysis of NUSAP1, CDK1, phospho- $\beta$ III tubulin (S172) and $\beta$ tubulin in MCF7, T47D and MDA-MB231 cell lines following treatment with increasing concentrations $(0,1,5$ and $10 \mu \mathrm{M})$ of $C 28$ for $48 \mathrm{~h}$. GADPH was used as loading control. Values represent the average of two experiments. c Western blotting of NUSAP1, CDK1, phospho- $\beta$ III tubulin (S172) and $\beta$ tubulin in MCF7, T47D and MDA-MB-231 cell lines following inhibition (siRNA) of LMTK3. GADPH was used as loading control. Values represent the average of two experiments. $\mathbf{d}$ Western blotting showing the effects of LMTK3 overexpression, using a pCMV6-LMTK3 plasmid, on NUSAP1 protein levels in MCF7, T47D and MDA-MB-231 cell lines following $48 \mathrm{~h}$ pre-treatment with $10 \mu \mathrm{M}$ C28. GADPH was used as loading control. Values represent the average of two experiments. e Western blotting analysis showing the effects of NUSAP1 overexpression, using a pCMV6-NUSAP1 plasmid, on CDK1, phospho-BIII tubulin (S172) and phospho-histone H3 (Ser10) in MCF7, T47D and MDA-MB-231 cell lines following $48 \mathrm{~h}$ pre-treatment with $10 \mu \mathrm{M}$ C28. GADPH was used as loading control. Values represent the average of two experiments. $\mathbf{f} L M T K 3$ or NUSAP1 were immunoprecipitated from MCF7 cells stably overexpressing LMTK3, and the complexes were immunoblotted for LMTK3 and NUSAP1. Western blots for the respective proteins in whole cell lysates (input) were also performed. $\mathbf{g}$ Kaplan-Meier plots (http://kmplot.com/) demonstrating the association of the mean expression of LMTK3 and NUSAP1 with overall survival in 626 BC patients. HR, hazard ratio. $\mathbf{h}$ Kaplan-Meier plots (http://kmplot.com/)

demonstrating the association of the mean expression of LMTK3 and NUSAP1 with disease free survival in 1764 BC patients

NUSAP1 correlates with shorter overall survival (Fig. 2g) and disease-free survival (Fig. 2h), indicating a plausible cooperation of LMTK3 and NUSAP1 in BC progression.

\section{Conclusions}

In the present study, we further demonstrated that $\mathrm{C} 28$ exerts its antitumor functions by degrading LMTK3 [2], which leads to a NUSAP1-mediated microtubule instability with subsequent cell-cycle arrest and cell death. Moreover, downregulation of NUSAP1-downstream proteins involved in cell cycle regulation and microtubule stability (CDK1 and phospho-BIII tubulin), after pharmacological and genetic LMTK3 inhibition, support this hypothesis (Supplementary Fig. S1b). Although we cannot rule out the possibility that the anticancer activity of $\mathrm{C} 28$ is also due to off-target effects, our LMTK3 and NUSAP1 recovery studies support that the observed results on microtubule stability are predominantly mediated via LMTK3 inhibition. In addition, the identification of NUSAP1 as a new interacting partner of LMTK3 suggests a direct interplay between LMTK3 and NUSAP1 pathways in the regulation of microtubule stability and validates the LMTK3 scaffolding properties, which can eventually contribute to tumorigenesis by enhancing signalling complexity [15]. Furthermore, our clinical data imply an association between NUSAP1 and LMTK3 in BC progression as shown by their unfavourable prognosis.

Combined, our findings propose that the pre-clinical therapeutic advantage of $\mathrm{C} 28$ stems from its effect on the LMTK3-targeted pathways linked to microtubule organization, acting differently from the established role of chemotherapeutic agents including vinca-alkaloids [16], taxanes [16] or eribulin [17], which confer their cytotoxicity via their interactions with tubulin causing disruption of microtubule function. The clinical use of microtubuletargeting drugs as anticancer drugs is well-established [18], while the ability of standard microtubule agents (i.e. nocodazole) to modulate the activities of certain kinases has also been reported [6]. Taken together, the development and design of multifunctional inhibitors can provide new, promising approaches for cancer treatment.

\section{Abbreviations}

LMTK3: Lemur tyrosine kinase 3; BC: Breast cancer; $K_{\mathrm{d}}$ : Dissociation constant; $\mathrm{NCl}$ : National cancer institute; ABL: Tyrosine-protein kinase ABL1; BRAF: Protooncogene BRAF; c-KIT: Proto-oncogene C-KIT; MEK: Mitogen-Activated Protein Kinase Kinase 1; TMT: Tandem mass tagging; NUSAP1: Nucleolar and spindle associated protein 1

\section{Supplementary Information}

The online version contains supplementary material available at https://doi. org/10.1186/s12943-021-01345-3.

Additional file 1: Supplementary Table S1. Identified proteins and comparative quantification of TMT label intensities.

Additional file 2: Supplementary Figure S1. Effects of C28 on microtubule organization in BC and non-transformed breast cell lines and schematic model depicting the proposed mechanism of action of C28 inhibitor. (a) MCF7, T47D, MDA-MB-231 and MCF12A cells were treated with $10 \mu \mathrm{M}$ of $\mathrm{C} 28$ for $48 \mathrm{~h}$. Cells were fixed and stained with anti-a-tubulin antibody (green) while the nuclear DNA was stained by DAPI (blue). Representative confocal microscopy images of mitotic phase cells are shown. Scale bar, 5 Mm. (b) C28 binds to LMTK3 promoting its proteasomemediated degradation. Downregulation of LMTK3 leads to a decrease in NUSAP1 and downstream proteins CDK1 and phospho- $\beta$ III tubulin (S172), resulting in cell cycle arrest, abnormal spindles and microtubules instability.

Additional file 3. Supplementary Materials and Methods.

\section{Acknowledgments}

We thank Dr Rosy Favicchio, Dr. Tom Stiff and Dr. Fabio Echegaray-lturra for the useful and stimulating discussions.

\section{Authors' contributions}

C.C. and A.D. performed most of the experiments and analysed the data. A.P. and G.M. conducted the tubulin binding experiments and docking calculations. T.G. performed the tubulin polymerization assays. M.E. and G.G. executed the TMT proteomics analyses. G.G. conceived the project and designed the study. J.S. reviewed and edited the manuscript. All authors read and approved the final manuscript. 


\section{Funding}

This work was supported by Action Against Cancer, The Colin McDavid Family Trust, The Helena Foundation, The Joseph Ettedgui Charitable Trust, Mr. Alessandro Dusi and The Cooper Family.

\section{Availability of data and materials}

The mass spectrometry proteomics data have been deposited to the ProteomeXchange Consortium via the PRIDE [19] partner repository with the dataset identifier PXD024644 and 10.6019/PXD024644. All the other data are available from the authors upon reasonable request.

\section{Declarations}

\section{Ethics approval and consent to participate}

Patient data we used were acquired by publicly available datasets that were collected with patients' informed consent.

\section{Consent for publication}

All authors give consent for the publication of the manuscript in Molecular Cancer. Supplementary Fig. S1b was created using biorender.com.

\section{Competing interests}

The authors declare that they have no competing interests.

\section{Author details}

${ }^{1}$ Department of Biochemistry and Biomedicine, School of Life Sciences, University of Sussex, JMS Building, Falmer, Brighton BN1 9QG, UK. ${ }^{2}$ National Centre for Scientific Research "Demokritos", Institute of Biosciences and Applications, 15341 Athens, Greece. ${ }^{3}$ Faculty of Medicine, Department of Surgery and Cancer, Imperial College, London W12 0NN, UK. ${ }^{4}$ Department of Medical Science, University of Udine, 33100 Udine, Italy.

Received: 30 October 2020 Accepted: 5 March 2021

Published online: 17 March 2021

\section{References}

1. Giamas $\mathrm{G}$, et al. Kinome screening for regulators of the estrogen receptor identifies LMTK3 as a new therapeutic target in breast cancer. Nat Med. 2011;17(6):715-9.

2. Angeliki Ditsiou, et al. The structure-function relationship of oncogenic LMTK3. Sci Adv. 2020;6(46):eabc3099.

3. Jordan MA, Wilson L. Microtubules as a target for anticancer drugs. Nat Rev Cancer. 2004:4(4):253-65.

4. Tanabe K, et al. Int J Mol Sci. 2017;18(12):2508.

5. Zhang C, et al. S9, a novel anticancer agent, exerts its anti-proliferative activity by interfering with both PI3K-Akt-mTOR signaling and microtubule cytoskeleton. PLoS One. 2009;4(3):e4881.

6. Park H, Hong S, Hong S. Nocodazole is a high-affinity ligand for the cancerrelated kinases ABL, c-KIT, BRAF, and MEK. ChemMedChem. 2012;7(1):53-6.

7. Lindamulage $\mathbb{K}$, et al. Novel quinolone chalcones targeting colchicinebinding pocket kill multidrug-resistant cancer cells by inhibiting tubulin activity and MRP1 function. Sci Rep. 2017;7(1):10298.

8. Ribbeck $K$, et al. A role for NuSAP in linking microtubules to mitotic chromosomes. Curr Biol. 2007;17(3):230-6.

9. Vanden Bosch A, et al. NuSAP is essential for chromatin-induced spindle formation during early embryogenesis. J Cell Sci. 2010;123(Pt 19):3244-55.

10. Chen $L$, et al. High levels of Nucleolar spindle-associated protein and reduced levels of BRCA1 expression predict poor prognosis in triplenegative breast Cancer. PLoS One. 2015;10(10):e0140572.

11. Sun L, et al. Overexpression of NuSAP1 is predictive of an unfavourable prognosis and promotes proliferation and invasion of triple-negative breast cancer cells via the Wnt/beta-catenin/EMT signalling axis. Gene. 2020;747:144657.

12. Zhang $X$, et al. Nucleolar and spindle associated protein 1 (NUSAP1) inhibits cell proliferation and enhances susceptibility to Epirubicin in invasive breast Cancer cells by regulating Cyclin D kinase (CDK1) and DLGAP5 expression. Med Sci Monit. 2018;24:8553-64.

13. Song Y, Brady ST. Post-translational modifications of tubulin: pathways to functional diversity of microtubules. Trends Cell Biol. 2015;25(3):125-36.

14. Wloga D, Gaertig J. Post-translational modifications of microtubules. J Cell Sci. 2010;123(Pt 20):3447-55.
15. $\mathrm{Xu} Y$, et al. LMTK3 represses tumor suppressor-like genes through chromatin remodeling in breast Cancer. Cell Rep. 2015;12(5):837-49.

16. Mukhtar E, Adhami VM, Mukhtar H. Targeting microtubules by natural agents for cancer therapy. Mol Cancer Ther. 2014;13(2):275-84

17. Dybdal-Hargreaves NF, Risinger AL, Mooberry SL. Eribulin mesylate: mechanism of action of a unique microtubule-targeting agent. Clin Cancer Res. 2015;21(11):2445-52.

18. Dumontet $C$, Jordan MA. Microtubule-binding agents: a dynamic field of cancer therapeutics. Nat Rev Drug Discov. 2010;9(10):790-803.

19. Perez-Riverol Y, Csordas A, Bai J, Bernal-Llinares M, Hewapathirana S, Kundu DJ, Inuganti A, Griss J, Mayer G, Eisenacher M, Pérez E, Uszkoreit J, Pfeuffer J, Sachsenberg T, Yılmaz S, Tiwary S, Cox J, Audain E, Walzer M, Jarnuczak AF, Ternent T, Brazma A, Vizcaíno JA. The PRIDE database and related tools and resources in 2019: improving support for quantification data. Nucleic Acids Res. 2019;47(D1):D442-D450.

\section{Publisher's Note}

Springer Nature remains neutral with regard to jurisdictional claims in published maps and institutional affiliations.
Ready to submit your research? Choose BMC and benefit from:

- fast, convenient online submission

- thorough peer review by experienced researchers in your field

- rapid publication on acceptance

- support for research data, including large and complex data types

- gold Open Access which fosters wider collaboration and increased citations

- maximum visibility for your research: over $100 \mathrm{M}$ website views per year

At BMC, research is always in progress.

Learn more biomedcentral.com/submissions 